\title{
Chimney Grafts in Acute Endovascular Aortic Repair Due to Ruptured Abdominal Aorta Aneurysm
}

\author{
Tal M Hörer
}

Department of Cardiothoracic and Vascular Surgery and Department of Surgery, Faculty of Medicine and Health, Örebro University Hospital and University, Sweden

Keywords: Aorta Aneurysm; Rupture; Chimney; Parallel Graft

These photos show how a chimney graft (parallel graft) was used in an urgent case of ruptured abdominal aortic aneurysm to facilitate endovascular aortic repair. Procedure time was around 90 minutes with complete recovery of the patient. The parallel graft technique was used due to very short aortic neck and landing zone. A GORE Excluder system and B-graft as chimney graft were used in this case.

\section{Corresponding author:}

Tal M Hörer, Department of Cardiothoracic and Vascular Surgery and Department of Surgery, Faculty of Medicine and Health, Örebro University Hospital and University, Sweden.

Email: tal.horer@regionorebrolan.se

(C) 2021 CC BY 4.0 - in cooperation with Depts. of Cardiothoracic/ Vascular Surgery, General Surgery and Anesthesia, Örebro University Hospital and Örebro University, Sweden

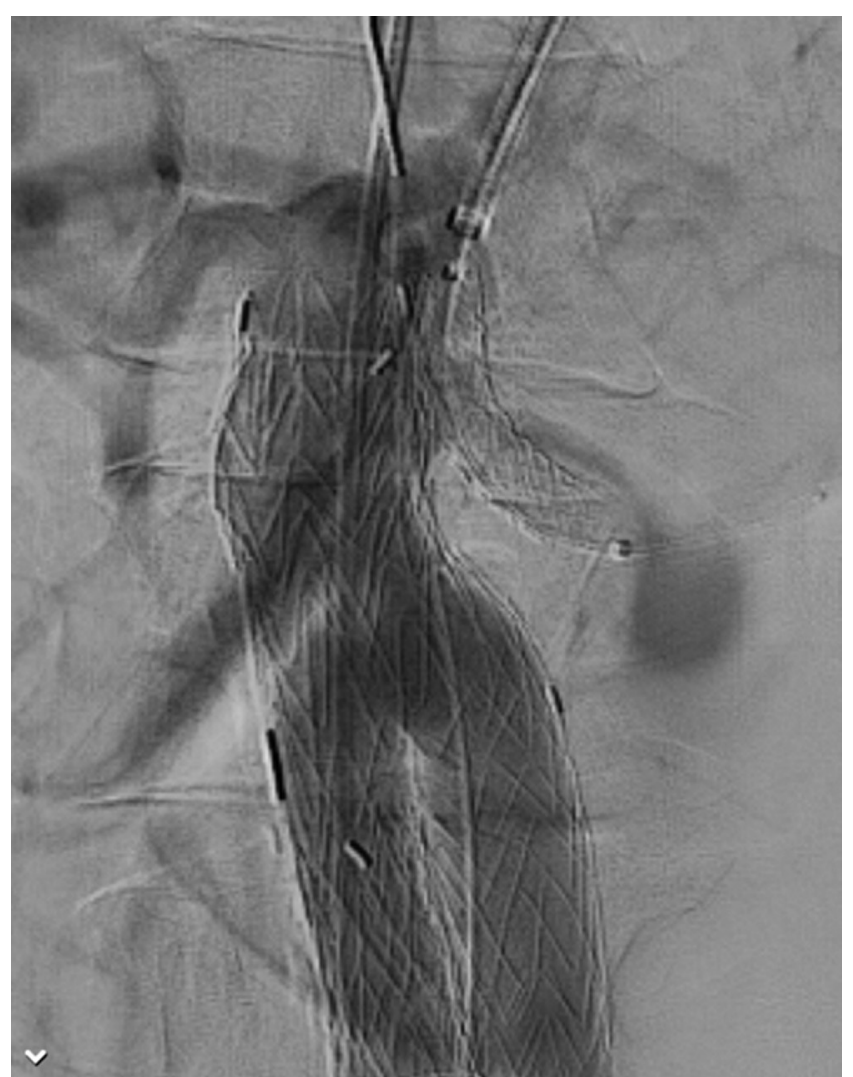

Figure 1 Peri-operative angiography showing the left kidney chimney graft position. 


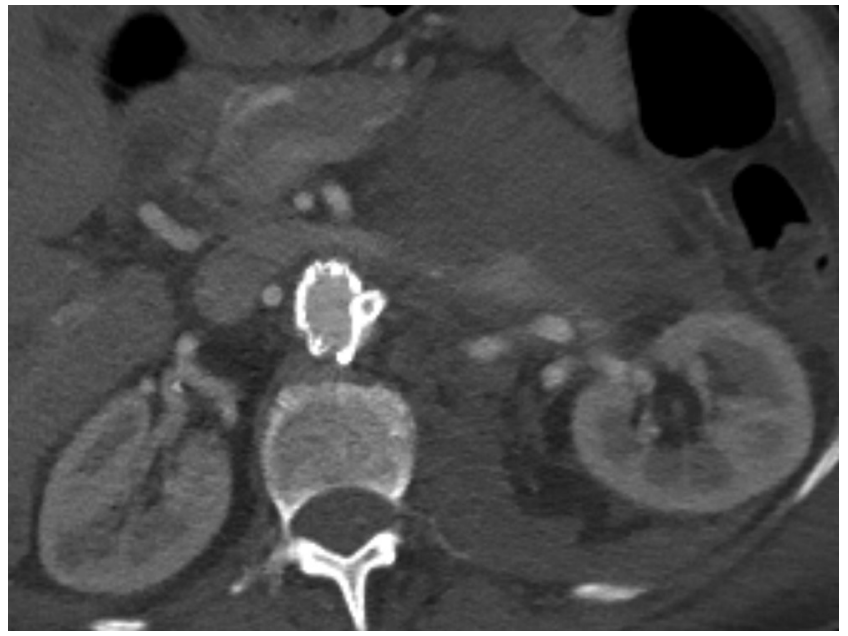

Figure 2 Axial view of computed tomography angiography (CTA) with chimney graft in the left kidney. No gutters seen and no endoleak on CTA.

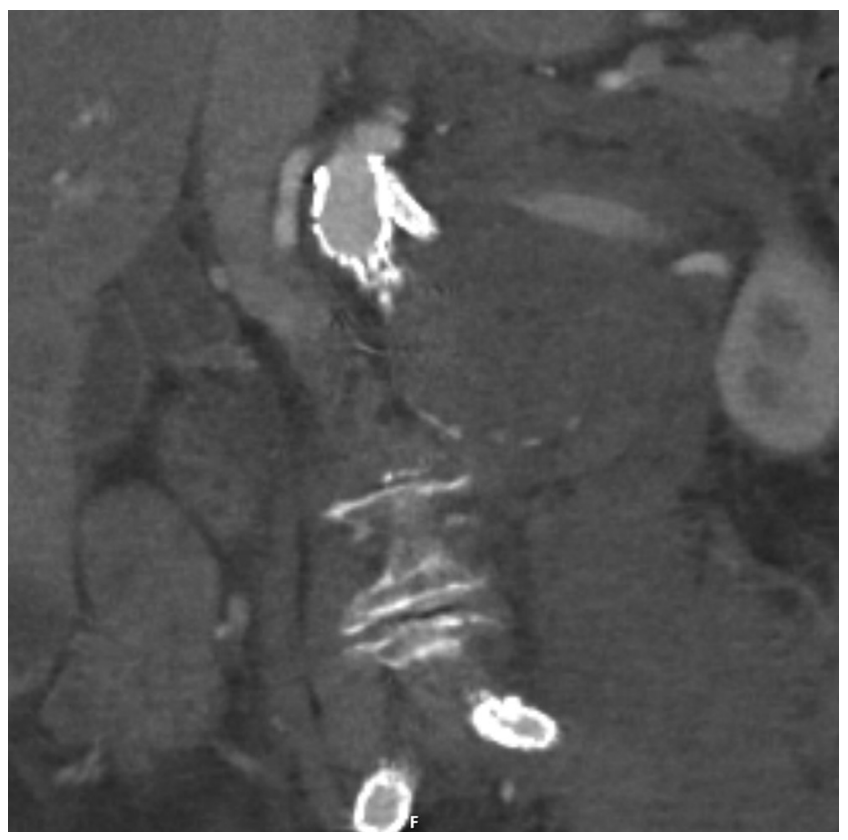

Figure 4 Coronal (frontal) view of the parallel graft on CTA.

\section{Ethics Statement}

(1) All the authors mentioned in the manuscript have agreed to authorship, read and approved the manuscript, and given consent for submission and subsequent publication of the manuscript.

(2) The authors declare that they have read and abided by the JEVTM statement of ethical standards including rules of informed consent and ethical committee approval as stated in the article.

(3) The images were published with permission from the patient.

\section{Conflicts of Interest}

The authors declare that they have no conflicts of interest.

\section{Funding}

The author received no financial support for the research, authorship, and/or publication of this article.
Figure 3 Axial view of the chimney graft parallel to the main graft body. 